\section{Chain Packing in Electro-Spun Poly(ethylene oxide) Visualized by Atomic Force Microscopy}

\section{R. J aeger, H. Schöherr, and G. J . Vancso*}

Faculty of Chemical Technology, University of Twente, P.O. Box 217, NL - 7500 AE Enschede, The Netherlands

Received J uly 19, 1996

Revised Manuscript Received September 3, 1996

Introduction. Scanning probe microscopy has been successfully used to investigate surfaces of polymers from the micrometer to the angstrom scale. ${ }^{1}$ With atomi c force microscopy (AFM), the profile of a polymer surface can be studied with molecular resolution. ${ }^{2}$ I mages revealed the packing of individual chains, and this packing was in agreement with the crystal structure of the polymer for a number of polymer systems. We used the AFM technique to examine the surface of poly(ethylene oxide) (PEO) $\left(\left[-\left(\mathrm{CH}_{2}\right)_{2}-\mathrm{O}-\right]_{n}\right)$ fibers and were able to obtain images of PEO macromolecular helices in the angstrom scale. The fibers were created with the electro-spinning process, in which a strong electrostatic field is used to form unusually thin fibers (the thinnest fibers which we obtained had a diameter of approximately $60 \mathrm{~nm}$ ) from a polymer solution.

Electrostatic spinning of fibers was first patented in the 1930s. ${ }^{3}$ Scientific studies of the electro-spinning process have been carried out by Baumgarten, ${ }^{4}$ Larrondo and Manley, ${ }^{5}$ and Reneker and co-workers. ${ }^{6}$ In particular, Doshi described the el ectro-spinning process for PEO fibers from aqueous solution. ${ }^{7}$

PEO usually crystallizes in a monoclinic phase where the polymer chains adopt a 7/2 helix. ${ }^{8}$ Stretching of the polymer results in a planar zigzag conformation with a triclinic crystal structure. ${ }^{9}$ Scanning probe microscopy was previously used to investigate the morphological details and surface structure of PEO crystals and poly(ethylene oxide)-polystyrene blends. ${ }^{10-12}$ Earlier studies on the nanometer scale focused on imaging chain folds on the surface of single crystals.

Experimental Section. We used PEO with an average molar mass of $2 \times 10^{6} \mathrm{~g} / \mathrm{mol}$ from Aldrich without further purification to prepare 1 wt \% aqueous solutions. The samples were gently stirred for $12 \mathrm{~h}$ at $40{ }^{\circ} \mathrm{C}$ to obtain homogeneous solutions. In order to electro-spin thin fibers, the PEO solution was first placed in a pipet. The air pressure above the solution was regulated with an air pump such that a drop of the PEO solution was suspended from the orifice of the pipet. We used a stainless steel pin immersed in the solution as an electrode. A flat metal plate (placed 7.5 $\mathrm{cm}$ below the pipet) was used as a grounded counter electrode. We connected the stainless steel pin to a Bertam 230-30R high-voltage supply which can generate dc voltages up to $30 \mathrm{kV}$. The applied voltage was displayed on the front panel of the Bertam high-voltage generator, and we determined the currents by placing a Hewlett Packard HP974A multimeter in series with the counter electrode and ground.

When we applied a voltage between the solution and the counter electrode, the pendant drop at the orifice of the pipet was deformed into a conical shape, called the "Taylor cone". ${ }^{13}$ At a threshold value of the surface charge density of the drop (for the experimental setup described above, the critical charge density was reached

* To whom correspondence should be addressed. at approximately $11 \mathrm{kV}$ ), the surface of the drop became unstable, and a jet was ejected from the cone. After the jet was formed, we reduced the voltage, thus making the jet narrower and increasing the charge density on the jet. At a certain voltage, the charge density on the jet reached a critical value where the jet splayed into several finer jets (at approximately $4 \mathrm{kV}$ and a current of $40 \mathrm{nA}$ ). A further reduction of the vol tage moved the splaying point of the jet toward the orifice. The jet ceased at a voltage of $3.5 \mathrm{kV}$. The voltages and currents stated in this section are system dependent (i.e. they vary with the type of polymer, the solvent, the concentration of the solution, the shape of the pipet, and the distance between the orifice and counter electrode).

In order to collect el ectro-spun fibers for light microscopy and AFM studies, we adjusted the voltage such that we obtained a stable jet which splayed approximately 5-10 $\mathrm{mm}$ bel ow the orifice. We collected the fibers on microscope slides for light microscope studies and on cover slides for AFM measurements. We placed the slides on the counter electrode in order to cover them with fibers. The fibers were examined without any further treatment.

The AFM measurements were carried out with a NanoScope III (Digital Instruments, Santa Barbara, CA) in the contact mode. AFM scans were performed in air, utilizing $\mathrm{Si}_{3} \mathrm{~N}_{4}$ cantilevers with a nominal spring constant of $0.38 \mathrm{~N} / \mathrm{m}$ (NanoTips, Digital Instruments). The whole system was allowed to equilibrate for $24 \mathrm{~h}$ prior to taking measurements. All images shown in this work correspond to unprocessed data. Most images were obtained in the so-called "height-mode"; some (where noted) were obtained in the friction mode. Images were captured at the same time with and without digital filtering (high pass set to 4, low pass set to 1). Angstrom scale periodicities were observed in both cases. Unless the images were excessively noisy, we used images which were obtained without filters for the subsequent analysis. In order to calibrate the instrument, mica was imaged after each experiment with the same setup (see below).

In order to obtain a quantitative analysis of the observed periodicities, we averaged the "up" and "down" scans. The images were analyzed by examining crosssectional plots perpendicular to the direction of the elongated periodic features. The repeat length of the periodic features was multiplied with a calibration factor (which takes into account the influence of sample height on measured distances and the fluctuation of the calibration of the instrument ${ }^{14,15}$ ). The angles of the periodic features were measured with respect to the fiber direction. We analyzed well over 100 images. The measured average is stated plus/minus the standard deviation.

An Olympus BX60 light microscope was used to investigate the birefringence of the electro-spun PEO fibers. Plots of the crystal structure of PEO were created with the Cerius $^{2}$ molecular modeling package. ${ }^{16}$

Results and Discussion. Most of the PEO fibers which were spun with the procedure described above (i.e. the jet ejected from the Taylor cone splayed approximately 5-10 $\mathrm{mm}$ from the orifice) exhibited a shape which resembled "beads on a string". We observed regularly spaced droplets of similar or alternating sizes al ong the fiber. The droplets were usually 1-5 $\mu \mathrm{m}$ wide; see Figure 1 . The diameter of the fibers typically ranged between 200 and $600 \mathrm{~nm}$. The thinnest fibers we observed were $60 \mathrm{~nm}$ wide. Droplet diameter 


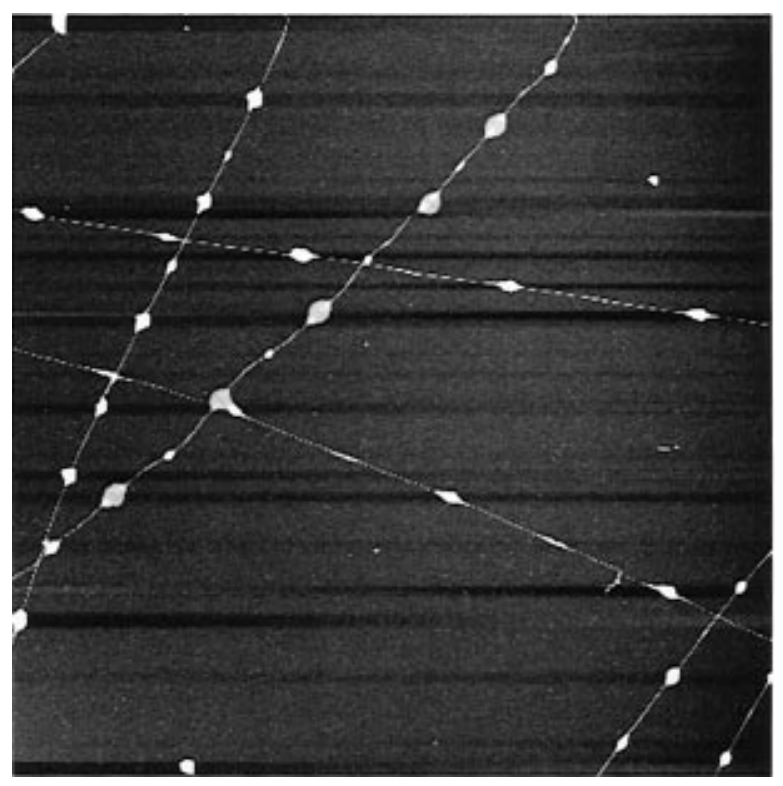

Figure 1. AFM image $(125 \mu \mathrm{m} \times 125 \mu \mathrm{m})$ of electro-spun PEO fibers. The fibers exhibit a "beads on a string" shape.

and spacing depended on the fiber diameter: the thinner the fiber, the smaller the distance between droplets and the diameter of the droplets.

A fiber which is spun from an aqueous solution can be regarded as a liquid cylinder which is supported along its axis. This type of cylinder is not stable; liquid flows spontaneously into evenly spaced droplets strung along the core fiber. ${ }^{17}$

The fibers between the droplets were birefringent when observed under crossed polars with an optical microscope. This indicates that the fibers were oriented. The droplets exhibited a pattern under crossed polars which resembled a "Maltese cross", indicating a radial orientation of the polarizability ellipsoids.

If we examine a surface area of approximately $10 \mathrm{~nm}$ $\times 10 \mathrm{~nm}$ of an electro-spun fiber with the AFM, the images reveal two sets of evenly spaced rows which form an angle of approximately $+50^{\circ}$ and $-50^{\circ}$ with the direction of the fiber axis. If the AFM tip scans parallel or perpendicular to the fiber axis, both sets of rows are simultaneously visible (see Figure 2a). If the tip samples the surface topology at an angle of $\pm 45^{\circ}$ to the fiber direction, only one of the sets of rows is visible-the one which is perpendicular to the scan direction. In Figure $2 b$ the rows are vertical, and in Figure $2 c$ the rows form an angle of $15^{\circ}$ with respect to the vertical direction. Since the scan directions of the two measurements are perpendicular to each other, the periodic features on the sample surface form an angle of $105^{\circ}$. This angle is approximately bisected by the fiber direction. We observed a spacing of $7.8 \pm 0.4 \AA$ for both sets of rows. These observations were repeated on several fibers.

Figure 3 shows the 100 facet of the monoclinic crystal modification of PE O. ${ }^{8}$ The PEO chains adopt a 7/2 helix in this crystal structure. The upper turns of the helices form ridges which are $7.8 \AA$ apart. The projection of the ridges is indined by $53^{\circ}$ to the crystallographic $c$ direction. The lower turns of the helices also form ridges with a spacing of $7.8 \AA$. The projection of these ridges forms an angle of $-53^{\circ}$ with the crystallographic c direction. The surface of the 010 facet of PEO results in a similar pattern. The top turns of the helices form ridges which are $7.9 \AA$ apart and are inclined by $55^{\circ}$ with respect to the crystallographic c direction, and the
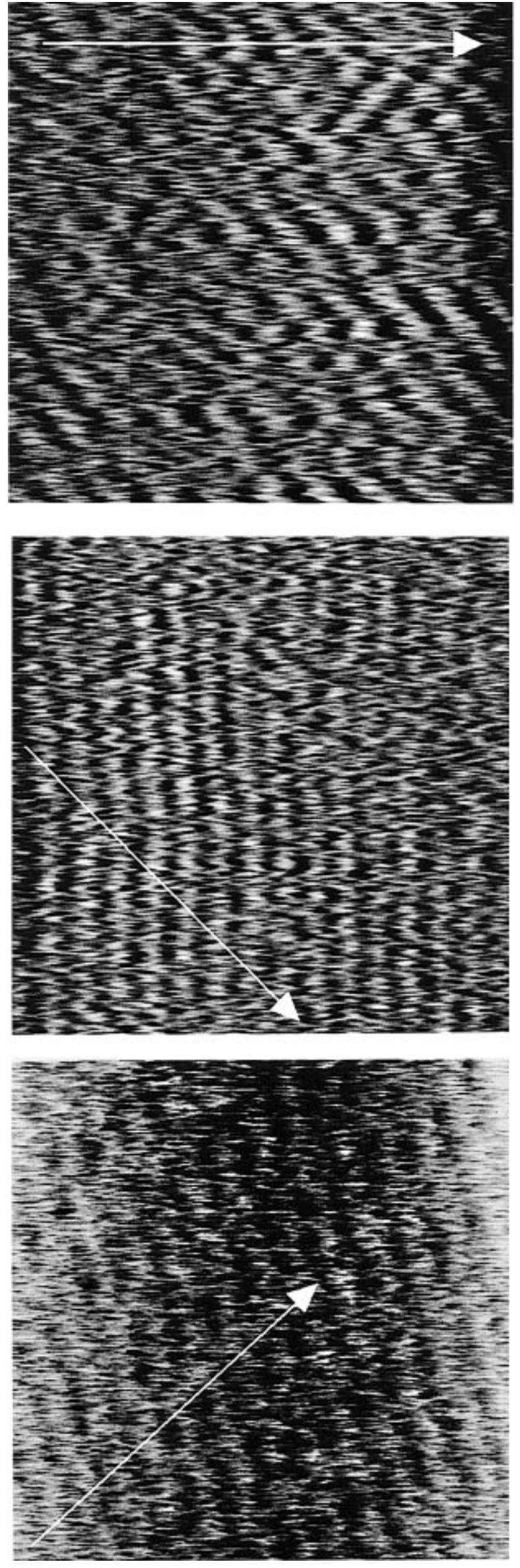

Figure 2. Nanographs of the surface of an electro-spun PEO fiber: (a) $11 \mathrm{~nm} \times 11 \mathrm{~nm}$ nanograph; (b) $12.5 \mathrm{~nm} \times 12.5 \mathrm{~nm}$ nanograph; (c) $12 \mathrm{~nm} \times 12 \mathrm{~nm}$ nanograph which was obtained in the friction mode. The white arrow indicates the fiber direction. The images show digitally unfiltered data. 


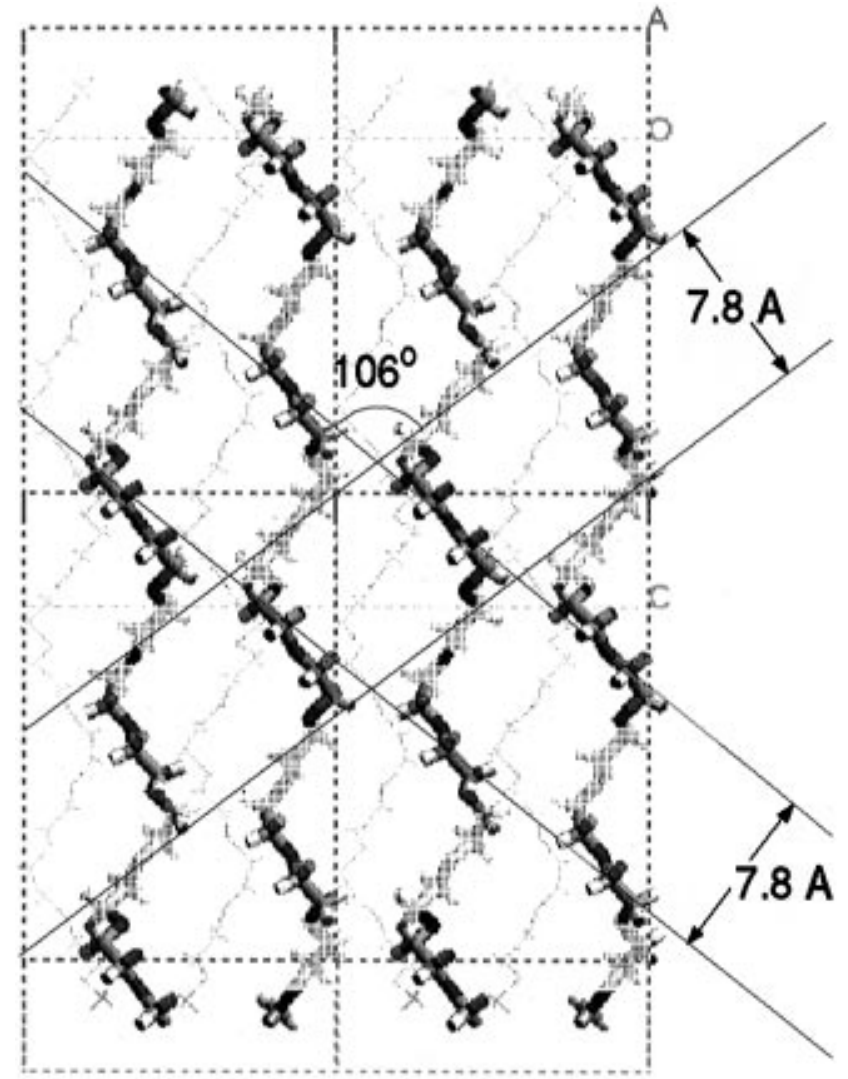

Figure 3. 100 facet of a PEO crystal. The top turns of the helices are drawn solid; the lower turns of the helices are shaded. Top and lower turns form ridges with an inclination of $\pm 53^{\circ}$ to the crystallographic c direction.

lower turns form ridges which are $7.7 \AA$ apart and form an angle of $-54^{\circ}$ with the crystallographic c direction.

The images we obtained with the atomic force microscope compare very well with the surface topology of the 100 or the 010 facet. We did not obtain any images which resembled other facets of PEO (e.g. the 120 facet and the -120 facet, which result in periodic structures with spacings of 6.5 and $6.9 \AA$ ). We ther efore identify the rowlike features which we observed in the images as top and bottom turns of helical PEO molecules in the top layer of the 100 or the 010 facet. Within the estimated accuracy of the instrument $\left( \pm 0.4 \AA\right.$ and $\left.\pm 4^{\circ}\right)$, it is not possible to distinguish between the two facets. If we compare the angle between the rows in the AFM images with the angle the ridges form with the crystallographic c direction, we see that the chain direction and the fiber direction are approximately parallel. The chain direction and the fiber direction both bisect the angle between the rows which is larger than $100^{\circ}$.
On the basis of our observations, we can conclude that at the molecular level the electro-spun PEO fibers possess a highly ordered surface layer. This ordered surface layer could be the result of the electro-spinning process, or it could be a residue of the dropl et formation. In order to determine the origin of this highly ordered surface layer, our future work will focus on the skincore structure of el ectro-spun fibers.

Acknowledgment. The financial support of Universiteit Twente is gratefully acknowledged. It is a pleasure to thank Ms. Anne Klemperer for her help with the preparation of the manuscript.

\section{References and Notes}

(1) (a) Binnig, G.; Quate, C. F.; Gerber, C. Phys. Rev. Lett. 1986, 56, 930. (b) The various imaging modes together with representative results obtained in polymer science are described in: Miles, M.J . Characterization of solid polymers: Chapman \& Hall: New York, 1994; pp 17-55. (c) For a recent comprehensive review see: Magonov, S. N.; Wangbo, M. H. Surface Analysis with STM and AFM; VCH: Weinheim, 1996. (d) For a review on the molecular architecture of polymer fibers see: Vancso, G. J. Polym. Prepr. (Am. Chem. Soc., Div. Polym. Chem.), 1996, 37 (2), 550.

(2) (a) Snétivy, D.; Vancso, G. J .; Rutledge, G. C. Macromolecules 1992, 25, 7037. (b) Snétivy, D.; Vancso, G. J . Colloids Surf. A 1994, 87, 257.

(3) Formhals, A. U.S. patent 2,116,942, 1938.

(4) Baumgarten, P. K.J . Colloid I nterfaceSci. 1971, 36 (1), 71.

(5) (a) Larrondo, L.; Manley, R. S. J . J . Polym. Sci., Polym. Phys. Ed. 1981, 19, 909. (b) Larrondo, L.; Manley, R. S. J.J. Polym. Sci., Polym. Phys. Ed. 1981, 19, 921. (c) Larrondo, L.; Manley, R. S. J . J . Polym. Sci., Polym. Phys. Ed. 1981, $19,933$.

(6) (a) Doshi, J .; Srinivasan, G.; Reneker, D. H. Polym. News 1995, 20, 206. (b) Doshi, J.; Reneker, D. H. J . Electrost. 1995, 35, 151. (c) Srinivasan, G.; Reneker, D. H. Polym. Int. 1995, 36 (2), 195.

(7) Doshi, J. The Electrospinning Process and Applications of Electrospun Fibers. Ph.D. thesis, University of Akron, Akron, $\mathrm{OH}, 1994$.

(8) Takahashi, Y.; Tadokoro, H. Macromolecules 1973, 6 (5), 672.

(9) Takahashi, Y.; Sumita, I.; Tadokoro, H.J . Polym. Sci., Part B: Polym. Phys. 1973, 11, 2113.

(10) Snétivy, D.; Vancso, G. J . Polymer 1992, 33 (2), 432.

(11) Motomatsu, M.; Nie, H.-Y.; Mizutani, W.; Tokumoto, H. Polymer 1996, 37 (1), 183.

(12) Nie, H.-Y.; Motomatsu, M.; Mizutani, W.; Tokumoto, H. J . Vac. Sci. Technol., B 1995, 13 (3), 1163.

(13) Taylor, G. I. Proc. R. Soc. London, A 1969, 313, 453.

(14) Snétivy, D.; Vancso, G. J . Langmuir 1993, 9, 2253.

(15) J aschke, M.; Schönherr, H.; Wolf, H.; Butt, H.-J .; Bamberg, E.; Besocke, M. K.; Ringsdorf, H. J . Phys. Chem. 1996, 100, 2290.

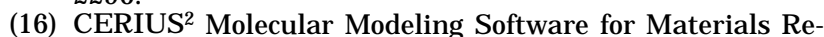
search from BIOSYM/Molecular Simulations Inc. of San Diego, CA, and Cambridge, U.K.

(17) Rayleigh, J. W. S. Philos. Mag. 1892, 34, 145.

MA9610673 